\title{
Manifestly covariant approach for the one-soliton sector *
}

\author{
H. O. Girotti ${ }^{\dagger}$ \\ Instituto de Física, Universidade Federal do Rio Grande do Sul, 90000 Porto Alegre, RS, Brasil \\ (Received 2 February 1977; revised manuscript received 11 April 1977)
}

\begin{abstract}
The one-loop contributions to the meson propagator and to the meson-soliton scattering amplitude in the one-soliton sector are analyzed using ordinary Feynman rules. It is found that, at the level of the propagator, the most severe infrared divergences cancel out while others remain. The structure of the remaining divergences is studied. We conclude that, among other things, the coefficient function of the leading divergence only depends upon the classical soliton solution. An extended Lehmann-SymanzikZimmerman reduction technique, to work in the one-soliton sector, is proposed. Then, it is shown that, under certain mathematical assumptions, the above-mentioned $S$-matrix element turns out to be free of zeromode singularities.
\end{abstract}

\section{INTRODUCTION}

As is well known, the computation of quantum corrections to properties of extended objects using the covariant functional approach faces a difficulty called the "zero-mode problem." One can avoid this difficulty by introducing collective coordinates, ${ }^{1}$ which of course implies a modification of the ordinary Feynman rules.

However, Faddeev and Korepin ${ }^{2}$ have explicitly computed the one- and two-loop contributions to the soliton self-energy using ordinary (manifestly covariant) Feynman rules, and they have found that, in this particular $S$-matrix element, all zeromode singularities cancel out. An extension of this result to all orders of the loop expansion has been carried out by Matveev. ${ }^{3}$

Nevertheless, the proposal of Faddeev and Korepin ${ }^{2}$ for dealing with the troublesome infrareddivergent part of the propagator has been criticized by Jevicki ${ }^{4}$ on the basis that it is ambiguous. Jevicki has carried out a consistent elimination of the zero-mode problem from the outset. The price to be paid for this advantage is that, besides the ordinary vertices coming from the action, there appears an additional set of vertices. This of course gives rise to a more complicated set of Feynman rules. The remarkable fact is that for the one- and two-loop contributions to the soliton self-energy, $Z_{1}$ and $Z_{2}$, respectively, one obtains the same result using either the Feynman rules derived by Jevicki ${ }^{4}$ or those proposed by Faddeev and Korepin. ${ }^{2}$ In the first case one talks about $\alpha$ independence ( $\alpha$ is a parameter related to the "amount" of translational symmetry breaking that is put into the theory in Jevicki's approach ${ }^{4}$ ) of $Z_{1}$ and $Z_{2}$, while in the second case one states that all zeromode singularities cancel out in $Z_{1}$ and $Z_{2}$. No proof has yet been presented concerning the equivalence of both covariant approaches as far as the contributions containing more than two closed loops are concerned. ${ }^{4}$

All computations we have mentioned so far refer to $S$-matrix elements, but no investigation has been carried out in order to see if a cancellation of zeromode singularities (or of $\alpha$-dependent terms) can also occur at the level of the Green's functions of the theory. This is precisely the first purpose of the present paper.

In Sec. II we compute the meson propagator in the one-soliton sector for a theory involving a real scalar field in a two-dimensional space-time. We shall always use the Feynman rules proposed by Faddeev and Korepin, ${ }^{2}$ but, since our calculations only involve one-loop corrections, it is easy to go from our results to the analogous ones which are obtained by using the approach of Jevicki. ${ }^{4}$ It is shown in this section that the most severe zeromode divergences cancel out while others remain. The remaining singular terms are properly isolated from the regular ones, and their structure is studied. Our results are analyzed in the light of the conclusions of Ref. 3 .

According to the proof supplied by Matveev in Ref. 3, which holds on general grounds, the $S$-matrix element associated with the Green's function that we found in Sec. II must be free of zero-mode singularities. One could of course use the procedure of Matveev $^{3}$ in order to check that, in particular, the meson-soliton scattering amplitude is free of infrared divergences. However, we have found it interesting to exhibit explicitly the mechanism through which the zero-mode divergences, which remain at the level of the connected two-point meson Green's function, vanish when the corresponding $S$-matrix element is computed.

To our knowledge, a formalism relating connected Green's functions and their corresponding $S$-matrix elements has not yet been developed for the one-soliton sector. In Sec. III we propose an 
extension, to the one-soliton sector, of the usual (vacuum sector) Lehmann-Symanzik-Zimmerman $(\mathrm{LSZ})^{5}$ reduction technique, which does the job. Then the process of elimination of infrared divergences arising when going from the Green's function to its corresponding $S$-matrix element can be properly studied.

Exhibiting the above-mentioned process, proposing a workable reduction formula for the one soliton-sector, and stating, with some precision, the mathematical assumptions under which an $S-$ matrix element free of zero-mode singularities arises are the remaining purposes of the present work.

The relevant points are discussed throughout the paper.

\section{THE MESON PROPAGATOR IN THE ONE-SOLITON SECTOR}

Our physical system is a two-dimensional scalar field theory described by the Lagrangian density

$$
\mathscr{L}=\frac{1}{2}\left(\partial_{\mu} \phi\right)\left(\partial^{\mu} \phi\right)-\mathfrak{u}(\phi) \text { 。 }
$$

Here $\phi$ is a real scalar field and $\boldsymbol{u}(\phi)$ is a Hermitian potential bounded from below. As usual, repeated Greek indices sum from 0 to 1 and our metric tensor $g_{\mu_{\nu}}$ is diagonal:

$$
g_{00}=-g_{11}=+1, \quad g_{\mu \nu}=0 \text { if } \mu \neq \nu .
$$

Let $\phi_{0}(x, a)$ be a confined (solitonlike) solution to the classical equations of motion which follow from (2.1). We denote by $a$ the coordinate of the center of mass of the soliton. For simplicity we shall assume that the field configuration $\phi_{0}$ is static. In fact, it is trivial to repeat the entire derivation which we shall present in this section for a timedependent solution.

When the theory is quantized around the classical solution $\phi_{0}$, one arrives, aside from numerical coefficients, at the following set of Feynman rules governing the loop expansion of the generating functional of connected Green's functions:

(a) A factor $\Delta\left(x, x^{\prime}\right)$ for each line joining the vertices $x$ and $x^{\prime}$.

(b) A factor $\mathfrak{u}^{(n)}(x)=\partial^{n} \mathfrak{u}(\phi) /\left.\partial \phi^{n}\right|_{\phi=\phi_{0}}$ for each $n$ line vertex at $x$.

(c) One is to integrate over all vertex coordinates.

The proposal of Faddeev and Korepin ${ }^{2}$ for dealing with the troublesome infrared divergence appearing in $\Delta$ is $[x \equiv(\tau, r)]$

$$
\begin{aligned}
\Delta\left(x, x^{\prime}\right) & =\Delta\left(\tau, r ; \tau^{\prime}, r^{\prime}\right) \\
& =c \psi_{a}(r) \psi_{a}\left(r^{\prime}\right)+\Delta_{0}\left(\tau, r ; \tau^{\prime}, r^{\prime}\right),
\end{aligned}
$$

where $c$ is a divergent constant and $\Delta_{0}$ is given by

$$
\Delta_{0}\left(\tau, r ; \tau^{\prime}, r^{\prime}\right)=-\frac{1}{2}\left|\tau-\tau^{\prime}\right| \psi_{a}(r) \psi_{a}\left(r^{\prime}\right)-\frac{1}{2} i \int_{-\infty}^{+\infty} \frac{d K}{\left(K^{2}+\mu^{2}\right)^{1 / 2}} \exp \left[-i\left(K^{2}+\mu^{2}\right)^{1 / 2}\left|\tau-\tau^{\prime}\right|\right] \psi_{K}(r) \psi_{K}^{*}\left(r^{\prime}\right)
$$

From the fact that the Lagrangian density (2.1) is translationally invariant (the potential $\mathcal{u}$ does not depend explicitly on $x$ ) while the classical solution $\phi_{0}(x ; a)$ is not, it follows that the operator $\hat{h}(r)$,

$$
\hat{h}(r)=-\frac{\partial^{2}}{\partial r^{2}}+u^{(2)}(r),
$$

must necessarily have a bound-state eigenfunction with zero eigenvalue. This eigenfunction is $\psi_{a}(r)=\partial \phi_{0}(x ; a) / \partial a$. Therefore,

$$
\hat{h}(r) \psi_{a}(r)=0 \text {. }
$$

The operator $\hat{h}$ could well have other bound states, sometimes known as excited states of the soliton, ${ }^{6}$ but we assume, as Faddeev and Korepin ${ }^{2}$ do, that no other bound states are present. This is of course the case of the sine-Gordon model. Furthermore, $\hat{h}$ has a continuum spectrum with eigenfunctions $\psi_{K}(r)$, i.e.

$$
\hat{h}(r) \psi_{K}(r)=\left(K^{2}+\mu^{2}\right) \psi_{K}(r),
$$

where $\mu$ is the meson mass, $K$ runs from $-\infty$ to $+\infty$, and the solutions $\psi_{K}(r)$ are interpreted as me- son-soliton scattering states. ${ }^{6}$

All quantities appearing in Eqs. (2.2) and (2.3) have already been defined. We note that from Eqs. (2.2) and (2.3) it follows that $\Delta\left(x, x^{\prime}\right)$ is a symmetric, although not a translationally invariant, function of its arguments.

As a final comment concerning the Feynman rules proposed in Ref. 2, we would like to remark that $\Delta_{0}$, given in (2.3), is a Green's function of the operator

$$
\begin{aligned}
H\left(x, x^{\prime}\right) & =\left[-\vec{\square}_{x}-\boldsymbol{u}^{(2)}(x)\right] \delta^{(2)}\left(x-x^{\prime}\right) \\
& =\left[-\frac{\partial^{2}}{\partial \tau^{2}}-\hat{h}(r)\right] \delta\left(\tau-\tau^{\prime}\right) \delta\left(r-r^{\prime}\right) .
\end{aligned}
$$

Obviously, $\Delta$ is the resolvent $(H+i \epsilon)^{-1}$ which becomes singular as $\epsilon \rightarrow 0$.

We turn next to the computation of the meson propagator $G\left(x, x^{\prime}\right)$ in the one-soliton sector retaining only up to one-loop corrections. By definition

$$
G\left(x, x^{\prime}\right)=-\left.i \frac{\delta^{2} Z[J]}{\delta J(x) \delta J(y)}\right|_{J=0},
$$


where $Z[J]$ is the generating functional of con nected Green's functions of the quantum theory and $J$ is an external source for the field $\phi$. The zero- and one-loop contributions to $G\left(x, x^{\prime}\right)$ are, respectively,

$$
\begin{aligned}
& G_{0}\left(x, x^{\prime}\right)=i \Delta\left(x, x^{\prime}\right), \\
& G_{1}\left(x, x^{\prime}\right)=-\frac{1}{2}\left[\int d^{2} y \int d^{2} y^{\prime} \Delta(x, y) \mathcal{u}^{(3)}(y) \Delta^{2}\left(y, y^{\prime}\right) \mathcal{u}^{(3)}\left(y^{\prime}\right) \Delta\left(y^{\prime}, x^{\prime}\right)+\int d^{2} y \Delta(x, y) \mathfrak{u}^{(4)}(y) \Delta(y, y) \Delta\left(y, x^{\prime}\right)\right. \\
& \left.\quad+\int d^{2} y \int d^{2} y^{\prime} \Delta(x, y) \mathcal{u}^{(3)}(y) \Delta\left(y, y^{\prime}\right) \mathcal{u}^{(3)}\left(y^{\prime}\right) \Delta\left(y^{\prime}, y^{\prime}\right) \Delta\left(y, x^{\prime}\right)\right] .
\end{aligned}
$$

The idea now is to study the $c$ dependence of $G_{0}$ and $G_{1}$ which is obtained by substituting (2.2) into (2.8) and (2.9). After some algebraic rearrangements we get

$$
\begin{aligned}
& G_{0}\left(x, x^{\prime}\right)=i c \psi_{a}(x) \psi_{a}\left(x^{\prime}\right)+i \Delta_{0}\left(x, x^{\prime}\right), \\
& -2 G_{1}\left(x, x^{\prime}\right)=A_{4}\left(x, x^{\prime}\right) c^{4}+A_{3}\left(x, x^{\prime}\right) c^{3}+A_{2}\left(x, x^{\prime}\right) c^{2}+A_{1}\left(x, x^{\prime}\right) c-2 G_{1}^{R}\left(x, x^{\prime}\right) .
\end{aligned}
$$

The expressions giving the coefficient functions $A_{i}\left(x, x^{\prime}\right), i=1,2,3,4$, are algebraically cumbersome, and we shall not pause to write them here. Clearly, the two-point function $G_{1}^{R}\left(x, x^{\prime}\right)$ is free of zero-mode divergences and it can be obtained from (2.9) by replacing $\Delta$ by $\Delta_{0}$.

Let us focus our attention on the divergent terms of $G_{1}\left(x, x^{\prime}\right)$. First of all, we mention that from the identities

$$
\begin{aligned}
& \int d^{2} y \cdot \mathfrak{u}^{(3)}(y) \psi_{a}{ }^{3}(y)=0, \\
& \int d^{2} y \mathfrak{u}^{(4)}(y) \psi_{a}{ }^{4}(y)+3 \int d^{2} y \mathfrak{u}^{(4)}(y) \psi_{a}{ }^{2}(y) \psi_{a a}(y)=0,
\end{aligned}
$$

derived by Faddeev and Korepin, ${ }^{2}$ one easily finds that

$$
\begin{aligned}
& A_{4}\left(x, x^{\prime}\right)=0, \\
& A_{3}\left(x, x^{\prime}\right)=0 .
\end{aligned}
$$

Here we have used $\psi_{a a}(y)$ as an abbreviated notation for $\partial \psi_{a}(y) / \partial a$.

Thus, all terms in $G_{1}$ proportional to $c^{4}$ and $c^{3}$ drop out. Then the leading singularity of $G_{1}$, induced by the infrared divergence of $\Delta$, is a simple pole $(1 / \epsilon)$ 。

We analyze next the terms proportional to $c^{2}$, i.e., $A_{2}\left(x, x^{\prime}\right)$ 。 Some terms contributing to $A_{2}\left(x, x^{\prime}\right)$ vanish partly because of $(2.12)$ and partly because the eigenvalues of $H$ do not depend on $a$. In fact, since the eigenvalues of $H$ do not depend on $a$ we can write that

$$
\frac{\partial^{n}}{\partial a^{n}}(\operatorname{tr} \ln H)=0, \quad n=1,2, \ldots
$$

In particular, for $n=1$ and $n=2$ we obtain from $(2.16)$ two identities which allow us to write the nonvanishing contributions to $A_{2}\left(x, x^{\prime}\right)$ as

$$
A_{2}\left(x, x^{\prime}\right)=\psi_{a}(x) \Omega\left(x^{\prime}\right)+\Omega(x) \psi_{a}\left(x^{\prime}\right)+\Lambda\left(x, x^{\prime}\right),
$$

where

$$
\begin{array}{r}
\Omega(x)=\int d^{2} y \Delta_{0}(x, y) \mathcal{u}^{(4)}(y) \psi_{a}{ }^{3}(y) \\
+3 \int d^{2} y \int d^{2} y^{\prime} \Delta_{0}(x, y) \psi_{a}(y) \mathcal{u}^{(3)}(y) \\
\quad \times \Delta_{0}\left(y, y^{\prime}\right) \psi_{a}{ }^{2}\left(y^{\prime}\right) \mathcal{u}^{(3)}\left(y^{\prime}\right)
\end{array}
$$

Our next step will be to show that the coefficient function $A_{2}\left(x, x^{\prime}\right)$ is fully controlled by the classical soliton solution $\phi_{0}(x)$.

Since the function $\psi_{a}(x)$ fulfills the homogeneous differential equation [see Eqs. (2.4) and (2.5)]

$$
\left[-\square-\mathfrak{u}^{(2)}(x)\right] \psi_{a}(x)=0,
$$

we conclude that

$$
\left[-\square-\mathfrak{u}^{(2)}(x)\right] \psi_{a a}(x)-\mathfrak{u}^{(3)}(x) \psi_{a}^{2}(x)=0,
$$

which when solved for $\psi_{a a}$ yields

$$
\psi_{a a}(x)=\int d^{2} y \Delta_{0}(x, y) \mathcal{u}^{(3)}(y) \psi_{a}{ }^{2}(y) .
$$

Then

$$
\begin{aligned}
\psi_{a a a}(x) \equiv \frac{\partial^{2} \psi_{a}(x)}{\partial a^{2}}= & \int d^{2} y \Delta_{0}(x, y) \mathcal{u}^{(4)}(y) \psi_{a}{ }^{3}(y) \\
& +\int d^{2} y \frac{\partial \Delta_{0}(x, y)}{\partial a} \mathcal{u}^{(3)}(y) \psi_{a}{ }^{2}(y)+2 \int d^{2} y \int d^{2} y^{\prime} \Delta_{0}(x, y) \mathcal{u}^{(3)}(y) \psi_{a}(y) \Delta_{0}\left(y, y^{\prime}\right) \mathcal{u}^{(3)}\left(y^{\prime}\right) \psi_{a}{ }^{2}\left(y^{\prime}\right) .
\end{aligned}
$$


To compute $\partial \Delta_{0} / \partial a$ we recall that $\Delta_{0}$ is a Green's function of $H$ and we obtain

$$
\frac{\partial \Delta_{0}\left(x, x^{\prime}\right)}{\partial a}=\int d^{2} y \Delta_{0}(x, y) \mathcal{u}^{(3)}(y) \psi_{a}(y) \Delta_{0}\left(y, x^{\prime}\right)
$$

in view of which $\psi_{a a a}(x)$ can also be written

$$
\psi_{a a a}(x)=\int d^{2} y \Delta_{0}(x, y) \mathcal{u}^{(4)}(y) \psi_{a}{ }^{3}(y)+3 \int d^{2} y \int d^{2} y^{\prime} \Delta_{0}(x, y) \mathcal{u}^{(3)}(y) \psi_{a}(y) \Delta_{0}\left(y, y^{\prime}\right) \mathcal{u}^{(3)}\left(y^{\prime}\right) \psi_{a}{ }^{2}\left(y^{\prime}\right)
$$
that

After comparing (2.18) with $(2.24)$ we conclude

$$
\Omega(x)=\psi_{\text {aaa }}(x),
$$

while from (2.19) and (2.22) it arises that

$$
\Lambda\left(x, x^{\prime}\right)=\psi_{a a}(x) \psi_{a a}\left(x^{\prime}\right) .
$$

By substituting (2.25) and (2.26) into (2.17) we arrive at the following final expression for $A_{2}\left(x, x^{\prime}\right)$ :

$$
\begin{aligned}
A_{2}\left(x, x^{\prime}\right) & =\psi_{a}(x) \psi_{a a a}\left(x^{\prime}\right)+\psi_{a a a}(x) \psi_{a}\left(x^{\prime}\right) \\
& +\psi_{a a}(x) \psi_{a a}\left(x^{\prime}\right)
\end{aligned}
$$

This result tells us that the coefficient function of the leading divergence is entirely determined by the eigenstate $\psi_{a}(x)$ of the operator $H$ or, what amounts to the same thing, that $A_{2}\left(x, x^{\prime}\right)$ only depends upon the classical soliton solution $\phi_{0}$, since $\psi_{a}=\partial \phi_{0}(x ; a) / \partial a$. We would like to remark also that each individual contribution to $A_{2}\left(x, x^{\prime}\right)$ is separable, i.e., it is of the form $f(x) g\left(x^{\prime}\right)$. This highly peculiar structure of $A_{2}\left(x, x^{\prime}\right)$ will play an important role in the cancellation of zero-mode divergences at the level of the $S$ matrix.

The next step of the present analysis consists in studying the coefficient function $A_{1}\left(x, x^{\prime}\right)$ which includes all terms multiplying the first power of $c$. After some algebra we have arrived at the following result:

$$
A_{1}\left(x, x^{\prime}\right)=\psi_{a}(x) \frac{\partial}{\partial a}\left|\frac{\delta}{\delta J\left(x^{\prime}\right)}\left(\operatorname{tr} \ln \Delta_{0}{ }^{-1}\right)\right|_{J=0}+\frac{\partial}{\partial a}\left|\frac{\delta}{\delta J(x)}\left(\operatorname{tr} \ln \Delta_{0}{ }^{-1}\right)\right|_{J=0} \psi_{a}\left(x^{\prime}\right)+\frac{\partial^{2} \Delta_{0}\left(x, x^{\prime}\right)}{\partial a^{2}}
$$

In the derivation of this last expression, the details of which are omitted for reasons of brevity, we have explicitly used the identity

$$
\begin{aligned}
\frac{\partial^{2} \Delta_{0}\left(x, x^{\prime}\right)}{\partial a^{2}} & =2 \int d^{2} y \int d^{2} y^{\prime} \Delta_{0}(x, y) \mathcal{u}^{(3)}(y) \psi_{a}(y) \Delta_{0}\left(y, y^{\prime}\right) \mathcal{u}^{(3)}\left(y^{\prime}\right) \psi_{a}\left(y^{\prime}\right) \Delta_{0}\left(y^{\prime}, x^{\prime}\right) \\
& +\int d^{2} y \Delta_{0}(x, y) \mathcal{u}^{(4)}(y) \psi_{a}{ }^{2}(y) \Delta_{0}\left(y, x^{\prime}\right)+\int d^{2} y \Delta_{0}(x, y) \mathcal{u}^{(3)}(y) \psi_{a a}(y) \Delta_{0}\left(y, x^{\prime}\right)
\end{aligned}
$$

which can be obtained by taking $\partial / \partial a$ on both sides of (2.29).

As one can see from (2.23), the entire spectrum of $H$ enters in the determination of $A_{1}\left(x, x^{\prime}\right)$. Furthermore, not all individual contributions to $A_{1}\left(x, x^{\prime}\right)$ are of a separable type.

The study of the coefficient functions of singular terms appearing in $G_{1}\left(x, x^{\prime}\right)$ is thus completed. We can now write down an expression for the meson propagator in the one-soliton sector, including up to one-loop corrections, in which all zero-mode divergences have been singled out. From Eqs. (2.10), (2.11), (2.14), (2.15), (2.27), and (2.28) one can easily see that

$$
\begin{aligned}
G\left(x, x^{\prime}\right)= & G_{0}\left(x, x^{\prime}\right)+\hbar G_{1}\left(x, x^{\prime}\right) \\
=\left(i c \psi_{a}(x) \psi_{a}\left(x^{\prime}\right)-\frac{1}{2} \hbar\right. & \left\{c\left[\psi_{a}(x) \frac{\partial}{\partial a}\left|\frac{\delta}{\delta J\left(x^{\prime}\right)}\left(\operatorname{tr} \ln \Delta_{0}^{-1}\right)\right|_{J=0}+\frac{\partial}{\partial a}\left|\frac{\delta}{\delta J(x)}\left(\operatorname{tr} \ln \Delta_{0}^{-1}\right)\right|_{J=0} \psi_{a}\left(x^{\prime}\right)+\frac{\partial^{2} \Delta_{0}\left(x, x^{\prime}\right)}{\partial a^{2}}\right]\right. \\
& \left.\left.+c^{2}\left[\psi_{a}(x) \psi_{a a a}\left(x^{\prime}\right)+\psi_{a a a}(x) \psi_{a}\left(x^{\prime}\right)+\psi_{a a}(x) \psi_{a a}\left(x^{\prime}\right)\right]\right\}\right) \\
& +i \Delta_{0}\left(x, x^{\prime}\right)+\hbar G_{1}^{R}\left(x, x^{\prime}\right)
\end{aligned}
$$

From a simple inspection of Eq. (2.30) it follows that, as we have already remarked, the most severe infrared divergences (i.e., terms proportional to $c^{4}$ and to $c^{3}$ ) cancel out in the expression for $G\left(x, x^{\prime}\right)$. Nevertheless, there still remain, in
$G\left(x, x^{\prime}\right)$, zero-mode singularities which cannot be absorbed by renormalizing $c$.

Thus, our conclusion is that there are closedloop contributions to $G\left(x, x^{\prime}\right)$ containing zero-mode singularities. This statement by no means implies 
that the above-mentioned divergences should be present in the meson-soliton scattering amplitude. In fact, we shall show in the next section that, under given mathematical assumptions, the $S$-matrix element associated with the Green's function $G\left(x, x^{\prime}\right)$ is independent of $c$.

This cancellation of zero-mode divergences at the $S$-matrix level has been interpreted by Matveev ${ }^{3}$ as an indication that an underlying symmetry, originally broken by a specific classical solution $\phi_{0}$, is being restored. Since the conclusion of Matveev ${ }^{3}$ seems to hold on quite general grounds (to each order in the loop expansion of the $S$ matrix), we are forced to recognize that there exists a significant difference between this restored symmetry and any internal symmetry that the theory might eventually possess. For example, there are no closed-loop contributions to the gauge-dependent part of the photon propagator in quantum electrodynamics, and we know that this property is intimately related to the gauge-invariant character of the corresponding $S$ matrix.

We close this section by mentioning that using the approach of Jevicki ${ }^{4}$ one obtains for $G\left(x, x^{\prime}\right)$, in the one-loop approximation, exactly the expression (2.30) with $c$ replaced by $\alpha$, where $\alpha$ is an arbitrary parameter whose inverse somehow measures the "amount" of translational symmetry breaking that is fed into the theory. As is obvious, for $\alpha=\infty$ one recovers, for $G\left(x, x^{\prime}\right)$, the result that is obtained by using the Feynman rules proposed by Faddeev and Korepin. ${ }^{4}$

\section{THE MESON-SOLITON SCATTERING AMPLITUDE}

We turn now to the analysis of the zero-mode singularity structure of the $S$-matrix element

$$
\left.S_{\alpha \beta}={ }_{\text {out }}\langle 1 \text { meson }| 1 \text { meson }\right\rangle_{\text {in }}
$$

in the one-soliton sector. We shall refer to $S_{\alpha \beta}$ as the meson-soliton scattering amplitude.

We could well follow Matveev ${ }^{3}$ and show that $S_{\alpha \beta}$ is free of infrared singularities. ${ }^{3}$ However, with this kind of technique it would not be possible to exhibit explicitly the process of elimination of infrared divergences which seems to occur when one goes from the Green's function to its corresponding $S$-matrix element. Since the purpose of this section is to study, at least in a particular case, the above-mentioned process, we must find a way to connect Green's functions and $S$-matrix elements in the one-soliton sector.

In the vacuum sector this problem is solved by the $\mathrm{LSZ}^{5}$ reduction technique, which allows one to conclude that the $S$-matrix element describing the process $n$ particles in the incoming channel

$$
\rightarrow m \text { particles in the outgoing channel }
$$

is just the Green's function for the $r=n+m$ particles with the external legs removed and with the external momenta put onto the mass shell. ${ }^{7}$ We conjecture that this result can be generalized to the one-soliton sector, and we therefore write for the transition $(T)$ matrix

$$
\begin{aligned}
& T_{\alpha \beta}=-\int d^{2} x \int d^{2} x^{\prime} f_{B}^{*}(x)\left[-\vec{\square}_{x}-u^{(2)}(x)\right] \\
& \times G\left(x, x^{\prime}\right)\left[-\mathbb{\square}_{x^{\prime}}-\mathcal{u}^{(2)}\left(x^{\prime}\right)\right] f_{\alpha}\left(x^{\prime}\right) .
\end{aligned}
$$

Here the operators $\left[-\square_{x}-\mathfrak{u}^{(2)}(x)\right]$ and $\left[-\square_{x^{\prime}}-\mathfrak{u}^{(2)}\left(x^{\prime}\right)\right]$ remove the two external meson legs, respectively, from the Green's function $G\left(x, x^{\prime}\right)$, while the $f_{\alpha}(x)$ 's form a complete orthogonal set of normalizable positive-energy solutions of the homogeneous equation

$$
\left[-\square-\mathfrak{u}^{(2)}(x)\right] f_{\alpha}(x)=0 .
$$

We want to stress that by "normalizable solution" we mean a square-integrable function over spacetime. As is obvious, the physical meaning of $f_{\alpha}(x)$ is that it represents an on-mass-shell state of a meson scattering from the soliton.

In connection with Eq. (3.2) we must say that we have found no difficulties in extending formally, to the one-soliton sector, the usual steps through which in the vacuum sector one is led to the reduction formula. The function $f_{\alpha}$ 's are required to satisfy the orthogonality conditions

$$
\begin{aligned}
& i \int_{-\infty}^{+\infty} d r f_{\alpha}^{*}(x) \vec{\partial}_{0} f_{\beta}(x)=\delta_{\alpha \beta}, \\
& i \int_{-\infty}^{+\infty} d r f_{\alpha}(x) \vec{\partial}_{0} f_{\beta}(x)=0
\end{aligned}
$$

in complete analogy with the conditions which must be obeyed, in the vacuum sector, by the wavepacket solutions of the free-field equation.

Let us concentrate our attention on those contributions to $T_{\alpha \beta}$ bearing zero-mode divergences. After (2.30) is replaced in (3.2) and account is taken of (2.20) we arrive at the conclusion that the only potentially dangerous ( $c$-dependent) terms remaining in the $T$ matrix are

$$
\begin{aligned}
& D_{\alpha B}^{(1)}=-\frac{1}{2} \hbar c \int d^{2} x \int d^{2} x^{\prime} f_{B}^{*}(x)\left[-\vec{\square}_{x}-\mathcal{u}^{(2)}(x)\right] \\
& \times \frac{\partial^{2} \Delta_{0}\left(x, x^{\prime}\right)}{\partial a^{2}}\left[-\widetilde{\square}_{x^{\prime}}-\mathfrak{u}^{(2)}\left(x^{\prime}\right)\right] f_{\alpha}\left(x^{\prime}\right), \\
& D_{\alpha B}^{(2)}=-\frac{1}{2} \hbar c^{2}\left|\int d^{2} x f_{B}^{*}(x)\left[-\vec{\square}_{x}-\mathfrak{u}^{(2)}(x)\right] \psi_{a a}(x)\right|^{2} .
\end{aligned}
$$

Thus, not all $c$-dependent terms appearing in 
(2.30) are automatically eliminated by the action of the operators removing the external legs from the Green's function. The terms quoted in Eqs。(3.4) remain. Our following task will be to show that
$D_{\alpha \beta}^{(1)}$ and $D_{\alpha \beta}^{(2)}$ are pure surface contributions to the $S$ matrix.

We start analyzing $D_{\alpha \beta}^{(1)}$ 。From Eq. (2.29) we find that

$$
\begin{aligned}
D_{\alpha B}^{(1)}=-\frac{1}{2} \hbar c & {\left[2 \int d^{2} x \int d^{2} x^{\prime} f_{B}^{*}(x) \mathcal{u}^{(3)}(x) \psi_{a}(x) \Delta_{0}\left(x, x^{\prime}\right) \mathcal{u}^{(3)}\left(x^{\prime}\right) \psi_{a}\left(x^{\prime}\right) f_{\alpha}\left(x^{\prime}\right)\right.} \\
+ & \left.\int d^{2} x f_{B}^{*}(x) \mathcal{u}^{(4)}(x) \psi_{a}^{2}(x) f_{\alpha}(x)+\int d^{2} x f_{B}^{*}(x) \mathcal{u}^{(3)}(x) \psi_{a a}(x) f_{\alpha}(x)\right] .
\end{aligned}
$$

On the other hand, from (3.3) we obtain

$$
\begin{aligned}
f_{\alpha a} & \equiv \frac{\partial f_{\alpha}(x)}{\partial a} \\
& =\int d^{2} x^{\prime} \Delta_{0}\left(x, x^{\prime}\right) \mathcal{u}^{(3)}\left(x^{\prime}\right) \psi_{a}\left(x^{\prime}\right) f_{\alpha}\left(x^{\prime}\right),
\end{aligned}
$$

in view of which we can rewrite (3.5) as

$$
D_{\alpha \beta}^{(1)}=-\frac{1}{2} \hbar c \frac{\partial N_{\alpha \beta}^{(1)}}{\partial a},
$$

where $N_{\alpha \beta}^{(1)}$ is defined as

$$
N_{\alpha \beta}^{(1)} \equiv \int d^{2} x f_{B}^{*}(x) \mathcal{U}^{(3)}(x) \psi_{a}(x) f_{\alpha}(x) .
$$

It is now a trivial matter to show that $N_{\alpha B}^{(1)}$ is a pure surface term. In fact,

$$
N_{\alpha \beta}^{(1)}=\oint d s^{\mu}\left[f_{\alpha a}(x) \partial_{\mu} f_{B}^{*}(x)-f_{B}^{*}(x) \partial_{\mu} f_{\alpha a}(x)\right],
$$

as proposed.

A similar line of reasoning leads us to

$$
\begin{aligned}
N_{\beta}^{(2)} & \equiv \int d^{2} x f_{\beta}(x)\left[-\vec{\square}_{x}-\mathcal{U}^{(2)}(x)\right] \psi_{a a}(x) \\
& =\oint d s^{\mu}\left[\psi_{a a}(x) \partial_{\mu} f_{\beta}(x)-f_{\beta}(x) \partial_{\mu} \psi_{a a}(x)\right],
\end{aligned}
$$

which of course guarantees that $D_{\alpha \beta}^{(2)}$ is also a pure surface contribution to the $S$-matrix element under analysis.

It is clear that whether zero-mode singularities are present at the $S$-matrix level only depends upon whether the integrals quoted in (3.9) and (3.10) vanish。 If all $f_{\alpha}$ 's are normalizable functions, in the sense already indicated, then (3.9) and (3.10) are vanishing integrals and, as a consequence, $S_{\alpha \beta}$ turns out to be free of zero-mode divergences. On the other hand, one should note that the cancellation of zero-mode singularities becomes ambiguous in the limit

$$
f_{\alpha}(x) \rightarrow \frac{1}{\sqrt{2 \pi}} \exp \left[i\left(K^{2}+\mu^{2}\right)^{1 / 2} \tau\right] \psi_{K}(r),
$$

where the functions $f_{\alpha}$ 's become non-normalizable.

Therefore, we conclude that the absence of zeromode divergences in $S_{\alpha \beta}$ can only be strictly proved, at least in the present case, if one assumes that the incoming and outgoing meson states are represented by square-integrable functions over space-time.

To conclude, we find it interesting to remark that Faddeev and Korepin ${ }^{2}$ have attributed the nonuniqueness of the propagator $\Delta$, characteristic of their approach, to the non-normalizable character of the function $\partial \phi_{0}(x: a) / \partial a$, in spite of the fact that it converges rapidly as $|r| \rightarrow \infty$. In a different but related context we have met with the same difficulty.

\section{ACKNOWLEDGMENTS}

I am greatly indebted to Professor Th. A. J. Maris and Professor B. Jouvet for many illuminating discussions. I have also enjoyed discussions with Professor V. Herscovitz and Professor H. Fanchiotti.

It is also a great pleasure to thank Professor $\mathrm{K}$. Symanzik for reading the manuscript and for many valuable discussions during his visit to our Institute.
*Work supported by Conselho Nacional de Desenvolvimento Científico e Tecnológico and Financiadora de Estudos e Projetos, Brasil.

$\dagger$ On leave of absence from Consejo Nacional de Investigaciones Cientificas y Técnicas de Argentina.

${ }^{1}$ C. Callan and D. Gross, Princeton report (unpublished);

J. L. Gervais and B. Sakita, Phys. Rev. D 11, 2943
(1975); J. L. Gervais, A. Jevicki, and B. Sakita, ibid. 12, 1038 (1975); J. Goldstone and R. Jackiw, ibid. 11, 1486 (1975); E. Tomboulis, ibid. 12, 1678 (1975);

N. H. Christ and T. D. Lee, ibid. 12 , 1606 (1975); J. L. Gervais and A. Jevicki, Nucl. Phys. B110, 93 (1976); B110, 113 (1976).

${ }^{2}$ L. D. Faddeev and V. E. Korepin, Phys. Lett. $63 \mathrm{~B}, 435$ 
(1976).

${ }^{3}$ V. A. Matveev, Nucl. Phys. B121, 403 (1977).

${ }^{4}$ A. Jevicki, Nuc1. Phys. B117, 365 (1976).

${ }^{5} \mathrm{H}$. Lehmann, K. Symanzik, and W. Zimmermann, Nuovo Cimento $\underline{1}, 205$ (1955).
${ }^{6}$ See, for example, R. Jackíw, lectures delivered at the XV Cracow School of Theoretical Physics, Zakopane, Poland, 1975 (unpublished).

${ }^{7}$ J. D. Bjorken and S. Drell, Relativistic Quantum Fields (McGraw-Hill, New York, 1965). 